\title{
LWIR quantum efficiency measurements using a calibrated MCT photodiode read by a Cryo-HEMT-based amplifier
}

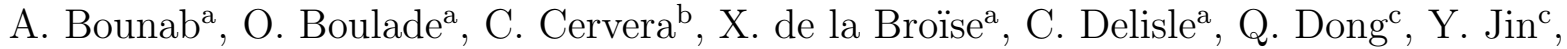 \\ A. Lamoure ${ }^{\mathrm{d}}$, L. Mathieu ${ }^{\mathrm{b}}$, V. Moreau ${ }^{\mathrm{a}}$, and P. Mulet ${ }^{\mathrm{a}}$ \\ ${ }^{a}$ Institut de Recherche sur les lois Fondamentales de l'Univers, CEA, Université Paris-Saclay, \\ F-91191Gif-sur-Yvette, France \\ ${ }^{b}$ Laboratoire Electronique et Traitement de l'Information, CEA Grenoble, 38054 Grenoble \\ France \\ ${ }^{\mathrm{c}} \mathrm{C} 2 \mathrm{~N}$, CNRS, Univ. Paris-sud, Univ. Paris-Saclay, 91120 Palaiseau, France \\ ${ }^{\mathrm{d}}$ Lynred, 38113 Veurey-Voroize France
}

\begin{abstract}
We present a new development for the measurement of the Quantum Efficiency (QE) of a Mercury Cadmium Telluride $(\mathrm{HgCdTe}$ or MCT) detector array in the long wave infrared (LWIR) spectral band. To measure the incident photon flux on the detector, CEA-LETI has designed and produced a calibrated MCT photodiode which, under the test setup conditions used for the QE measurement, delivers a total (dark plus photonic) current of $1 \mathrm{nA}$ at $60 \mathrm{~K}$. The readout of such a low level of current makes a standard room temperature amplifier inconvenient due to the length of the wires between the focal plane (FP) at cold and the outside of the cryostat $(>2 \mathrm{~m}$ in the current cryostat). A much better approach is to use High Electron Mobility Transistors (Cryo-HEMTs), optimized by CNRS/C2N laboratory for ultra-low noise at very low temperatures $(<1 \mathrm{~K})$. We have developed a Cryo-HEMT-based transimpedance amplifier to readout the photonic current of the calibrated MCT chip. The paper describes the calibrated photodiode, the Cryo-HEMT amplifier and the test setup, and shows the results of the QE measurements of the LWIR detector.
\end{abstract}

Keywords: Quantum Efficiency, Cryogenic Electronics, Cryo-HEMT, MCT, IR

\section{INTRODUCTION}

In the frame of its Cosmic Vision program, the European Space Agency (ESA) has started a number of technological development activities in the field of infrared detectors based on Mercury Cadmium Telluride ( $\mathrm{HgCdTe}$ or MCT) material. Since 2013, several activities have been conducted at CEA in the long wave infrared (LWIR) spectral range, with detector at a cutoff wavelength of $12.5 \mu \mathrm{m}$ at $40 \mathrm{~K}$. Similar developments have been funded by the French Space Agency (CNES) as part of the French contribution to the study of the Exoplanet Characterization Observatory (EChO) mission (which was proposed for ESA's medium mission M3 slot but was not selected). Results of these activities have been published in references ${ }^{1}$ and. ${ }^{2}$ More recently, Lynred has been leading a collaboration with CEA in a second ESA funded detector development in the same spectral range for future missions.

In this Lynred-CEA collaboration, CEA's Laboratoire Electronique et Traitement de l'Information (LETI) and Lynred manufactured several devices, $640 \times 512$ pixels in size at a $15 \mu \mathrm{m}$ pitch. CEA's Institute of Research into the Fundamental Laws of the Universe (IRFU) was in charge of the characterization of the detectors. As part of this activity, CEA also developed the test means required to assess the electro-optical performances (quantum efficiency, dark current, readout noise, linearity, dynamic range, ...) of these devices in the $20 \mathrm{~K}$ to $80 \mathrm{~K}$ range of temperatures.

Further author information:

A. Bounab: E-mail: ayoub.bounab@cea.fr, Telephone: +33 (0)1 69082228

O. Boulade: E-mail: olivier.boulade@cea.fr, Telephone: +33 (0)1 69085144

X-Ray, Optical, and Infrared Detectors for Astronomy IX, edited by Andrew D. Holland, James Beletic, Proc. of SPIE Vol. 11454, 1145428 - (c) 2020 SPIE

CCC code: $0277-786 \mathrm{X} / 20 / \$ 21 \cdot$ doi: $10.1117 / 12.2561458$

Proc. of SPIE Vol. 11454 1145428-1 
The quantum efficiency of a semi-conductor detector is its overall capability to absorb light and then collect the electrical charges generated by this absorption. It is basically the ratio of the current delivered by the detector (in e-/s/pixel) divided by the photon flux of the incident light illumination (in photon/s/pixel), and the difficulty is to accurately measure this photon flux falling at the surface of the detector.

The classical way of measuring the QE in the LWIR range is to first measure a relative spectral response using a Fourier Transform InfraRed spectrometer, by comparison to a reference detector in the FTIR. In a second step, an absolute QE measurement is performed using a calibrated light source (black body). One of the drawback of this technique is that, even though the photon flux emitted by the light source is known thanks to its calibration, the photon flux at the level of the detector is not known, unless one very accurately measures what happens along the light beam (absorption of photons by water vapor if the light path is in the air, transmission of the entrance window of the detector cryostat,...).

The technique presented in this paper is fundamentally different in that it simultaneously records the photon flux and the photonic current. This requires that a calibrated reference detector is placed somewhere in the light beam, and that the ratio of the photon fluxes at both locations (the reference detector and the detector under test) is accurately known. Provided these two requirements are met, neither the photon flux emitted by the light source nor the photon losses along the light path need to be known.

This second technique of measuring the QE is widely used in the visible spectral range, where calibrated Silicon photodiodes are commercially available. Unfortunately, there are no commercially available calibrated reference detector in the LWIR range. CEA-LETI therefore developed such a device, thanks to a funding from the French FOCUS Laboratory of Excellency. This development is described in section 2.

The photonic current delivered by the calibrated detector is typically about $1 \mathrm{nA}$. Given the length of the cryoharness inside the cryostat (more than $2 \mathrm{~m}$ ), it is very difficult to drive such a small current to the outside world. Therefore CEA-IRFU developed a cryogenic amplifier based on High Electron Mobility Transistors (Cryo-HEMTs), described in section 3.

Section 4 describes the test setup and the QE measurements on a first detector. Last, the perspectives of such developments are given in the conclusion.

\section{DEVELOPMENT AND CALIBRATION OF A REFERENCE LWIR MCT PHOTODIODE}

Thanks to its versatility in band gap and its capability to address the low flux conditions, HgCdTe photodetectors have been largely studied especially for space applications. Few years ago, constant quantum efficiency was demonstrated at LETI from the long wavelength infrared down to visible range. ${ }^{3}$ This part presents and discusses how we used MCT detector capability to detect visible light to calibrate the quantum efficiency.

\subsection{LWIR HgCdTe photodiode:}

In this work, the study photodiodes, with a cut-off wavelength close to $11.5 \mu \mathrm{m}$ at $80 \mathrm{~K}$, are based on p-on-n architecture. This architecture relies on a MCT active base layer grown by horizontal-slider LPE in rich-Te solution. This process grants a very flat epitaxial layer with a homogenous $\mathrm{xCd}$ cadmium composition. These points are especially critical in the LWIR and VLWIR spectral band as a small variation in Cd composition, typically $1 \%$, will result in a cutoff wavelength variation between 0.3 to $0.5 \mu \mathrm{m}$. MCT epitaxy is performed on a lattice matched CdZnTe with a Zn proportion depending on $\mathrm{Cd}$ composition and varying in the range of $1 \%$ to $5 \%$ from SWIR to VLWIR.

The n-type doping of the active base layer is obtained by extrinsic doping. Indium is used in this absorbing layer as a donor. Incorporation is done during epitaxy and In is naturally activated in MCT. In concentration varies from $5 * 10^{14}$ to $10^{16} \mathrm{at} / \mathrm{cm}^{3}$, and it has a direct influence on minority carrier lifetime. Thus it impacts carrier diffusion length and dark current. It has been shown that depending on the In concentration, minority carrier recombination process can change from Auger 1 to SRH, leading to different levels of dark current. ${ }^{4}$ This study allows determining the best In concentration accordingly to the structure bandgap and the other growth parameters.

p-on-n photodiodes are manufactured by ion-implantation of Arsenic. Two steps of annealing after implantation are necessary: one to repair defects resulting from ion bombardment and activate As and the other one, under $\mathrm{Hg}$ overpressure vapor, to restore n-type doping of the base layer. Hall and MEMSA (Maximum Entropy Mobility 
Spectrum Analysis $)^{5}$ measurements show that As activation rate is greater than $50 \%$. Finally the MCT surface is passivated, an electrical contact is taken and an anti-reflecting coating may be deposited on the backside of the CZT substrate (figure 1).

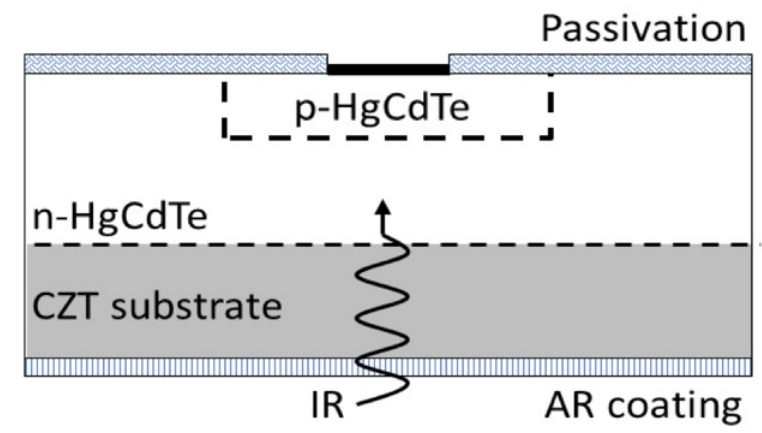

Figure 1. Cross sectional view of a p-on-n As-implantation planar device.

In this architecture, the minority carriers (hole) life time and mobility in the n-type layer lead to a lower dark current and a higher quantum efficiency than standard n-on-p architecture. That is why we decided to use it to develop a calibrated IR device.

\subsection{QE calibration method}

The calibration was two-staged. Phase 1 consisted of assessing the spectral responses. These measurements have been performed on two different spectrometers:

- A Fourier-Transform Infra-Red (FTIR) spectrometer continuously modulated with a Deuterated TriGlycene Sulfate (DTGS) as reference cell (thermal detector with a flat behavior as a function of the wavelength) and a choice of several sources as a function of the desired spectral range. Two possible Beam Splitters (BS) are also available: a CaF2 BS for $\lambda<7 \mu \mathrm{m}$ and a $\mathrm{KBr}$ BS for $1.5 \mu \mathrm{m}<\lambda<16 \mu \mathrm{m}$.

- A monochromator composed of xenon lamp, a rotating grating and a silicon calibrated (NIST calibration) photodiode covering visible and near infrared domain $(0.4$ to $1.1 \mu \mathrm{m})$. The light signal is modulated at $20 \mathrm{~Hz}$ with a mechanical chopper to obtain a high signal to noise ratio with a look-in amplifier.

At the beginning of the measurement campaign, a reference spectrum of the source is taken with the reference detector for both FTIR and monochromator. For example in FTIR configuration, the measured spectral response is given by the ratio between the measured diode spectrum and the reference spectrum taken from the DTGS. The output of the thermal reference detector is a power and the output of the characterized photodiode is a current, the resulting spectral response is homogenous to $\mathrm{A} / \mathrm{W}$. With the monochromator the same method has been used, but, thanks to two ports integrating sphere, the reference measurement and $\mathrm{HgCdTe}$ response can be recorded simultaneously. The source spectrum is then taken out from the reference silicon diode measurement, so response is the ratio between source spectrum and photodiode.

As all the $\mathrm{HgCdTe}$ photodiode measurements have been performed in variable cryostat temperature (from $80 \mathrm{~K}$ to $50 \mathrm{~K}$ ), the transmittance spectrums of cryostat windows were also measured. Figure 2 shows the results for quartz (visible) and polished ZnSe (IR) windows used to correct diode spectral response. All the response spectrums in this paper have been adjusted by window transmittances. 


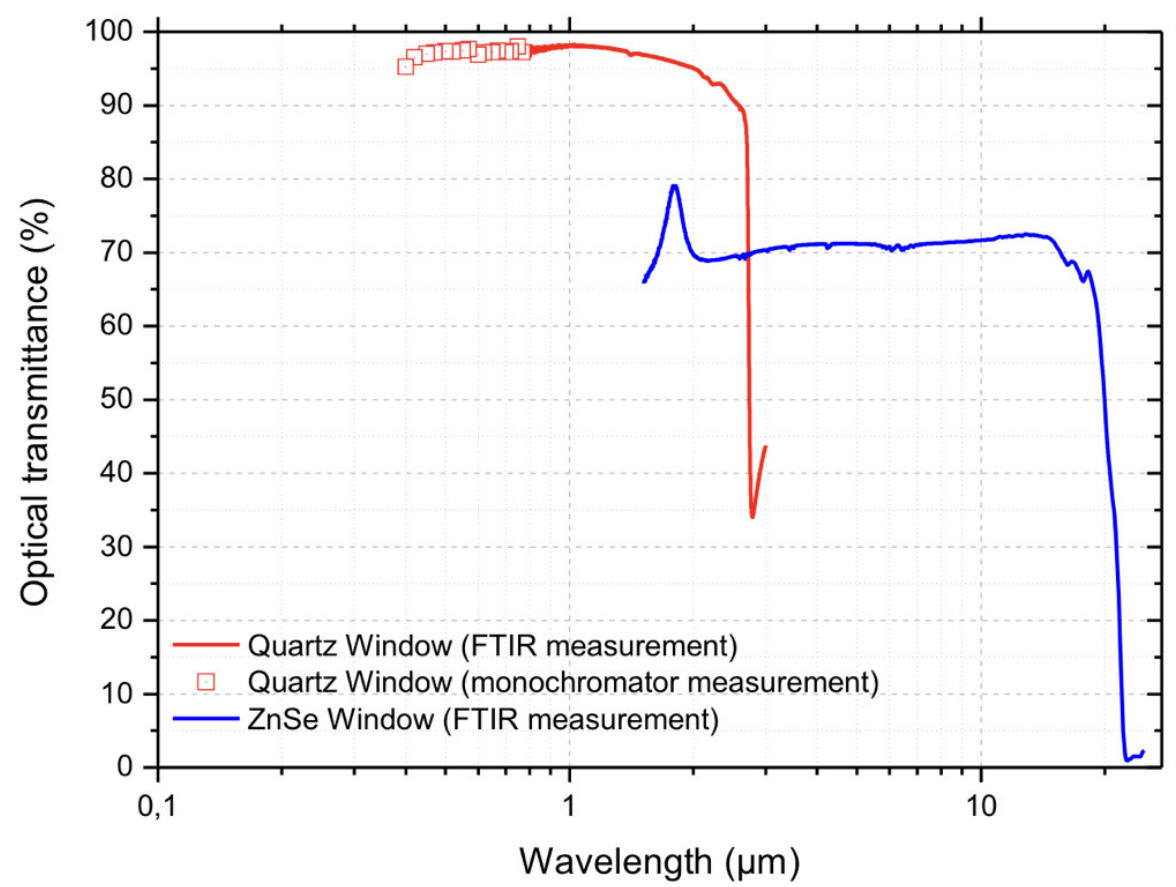

Figure 2. Optical Transmittances of quartz and ZnSe cryostat windows.

In a second step, quantum efficiency was calibrated in both visible and IR domains.

- In visible range the experimental set-up is exactly the same than spectral response. Actually, from the same measurement we record simultaneously the silicon photodiode and HgCdTe detector signals. As explained before we obtain source spectral emittance in Watt $(\mathrm{W})$, knowing silicon calibrated response. Then, taking in account geometrical ratio (field of view and diode size) between reference diode (FOV $=2 \pi$, size $1 \mathrm{~cm}^{2}$ ) and $\mathrm{HgCdTe}$ detector (FOV=30deg, $30 \mu \mathrm{m}$ pitch), we quantify HgCdTe QE. Obviously, before making such calculations we check the axial symmetry of the integrating sphere, providing power measurement on the two outputs. The difference is around $2 \%$.

- QE in IR wavelengths was estimated using a hot cavity Black-Body (BB) chopped at 30Hz. The output signal of the detector under test is the value of the mean harmonic ( $\mathrm{Sf}$ ) corresponding to the chopper modulation. This signal corresponds to the differential response between the BB cavity temperature and the chopper blade. The photon flux is defined by the extent between the diaphragm (area Adiaph) and detecting area pixel (Apix) separated by a distance d. Given the fact that the chopped BB signal is a square signal between high temperature cavity emittance $\left(T_{B B}\right)$ and ambient temperature (T0), the BB response is given by:

$$
R_{1-B B}\left(T_{B B}\right)=\frac{S_{f}}{\frac{\sigma}{\pi}\left(T_{B B}^{4}-T_{0}^{4}\right)} \frac{\pi / \sqrt{2}}{\frac{A_{D i a p h} * A_{p i x}}{d^{2}}}
$$

Where $\pi / \sqrt{2}$ is a corrective factor taking in account the square shape of the signal, and $\sigma$ is the Stefan constant. Moreover we calculate the ratio between spectral response at peak wavelength, $\operatorname{RI}\left(\lambda_{p}\right)$, and $\mathrm{BB}$ response as described above corresponding to the BB cavity temperature, thanks to the knowledge of the normalized spectral response alone $R_{I-N}\left(\lambda_{p}\right)$. This ratio is called $1 / \mathrm{G}$ and is described below:

$$
1 / G=\frac{R_{1}\left(\lambda_{p}\right)}{R_{1-B B}\left(T_{B B}\right)}=\frac{\sigma T_{B B}^{4}}{\int_{0}^{\text {inf }} R_{1-N}\left(\lambda_{p}\right) * E_{\lambda}\left(T_{B B}\right) d \lambda}
$$

Where $E_{\lambda}\left(T_{B B}\right)$ is the $\mathrm{BB}$ spectral emittance given by classical Planck equation. The knowledge of this factor allows the calculation of the QE at peak wavelength, and also the QE spectrum. 
The BB temperatures used for this study were $873 \mathrm{~K}$ and 523K. We also estimated QE precision taking into account geometrical aspect (diaphragm, distance) and cavity temperature. Altogether the final accuracy on QE should be close to $7 \%$.

\subsection{Results and discussion}

The key objective to this calibration is to use the silicon photodiode as a reference and compare the result with the IR measurements. As mentioned earlier, we benefit from the capability of HgCdTe material to keep a constant QE from UV to IR to do that. The photodiodes used at LETI are flip-chipped onto a Si fan-out for signal acquisition. In this configuration, incident light comes through the substrate (backside illumination). Therefore, without substrate removal the cut-on wavelength of the diode is defined by the substrate absorption $(0.8 \mu \mathrm{m}$ for $\mathrm{CdZnTe}$ ) whereas the cut-off is determined by the $\mathrm{HgCdTe}$ band gap. So, to achieve visible measurements, a chemical etching of the substrate was performed after hybridization on Si fan-out. Figure 3 presents the spectral responses measurements for a sample with and without substrate.
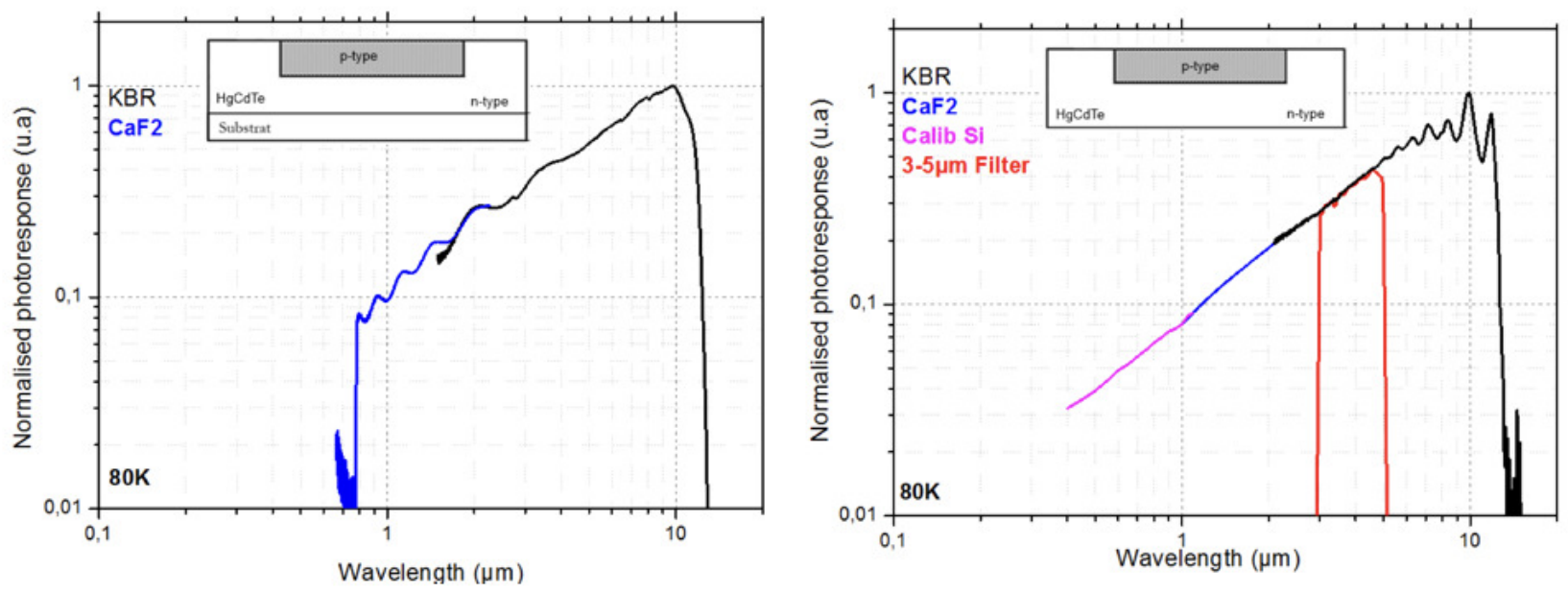

Figure 3. Normalized spectral response with the substrate (left curve) and without the substrate (right curve). Measurements were performed with different configurations to cover all the spectral range. FTIR measurements with KBR and CaF2 beam splitters and monochromator with calibrated Silicon photodiode.

Several differences are observed. In the first instance, we note that the measurement with substrate exhibits a cut-on wavelength close to $0.8 \mu \mathrm{m}$. It is the signature of substrate absorption. This effect is not observed with the thinning layer, and the measurement limit is only determined by monochromator grating $(0.4 \mu \mathrm{m})$. The cut-off is $11.6 \mu \mathrm{m}$ and is the same for the both curves. We observe also interferences fringes near the cut-on for the measurement with substrate and close to the maximum response for the measurement without substrate. These interferences are due to the optical cavity between the reflecting rear interface and the metalized contact on the other side of the absorbing layer. Since the sample thicknesses are not equal $(\mathrm{HgCdTe}+$ substrate vs $\mathrm{HgCdTe}$ only) the interferences do not occur at the same wavelengths on the spectrum responses. An efficient way to suppress this effect could be to deposit an anti-reflect coating on the incident light interface. However, we know that this operation on a small chip, $8 \mathrm{~mm}^{2}$ here, can generate a large mechanical stress, leading to separation between photodiodes and silicon fan-out, and some side effect on the thickness of AR-coating, leading to a non-uniform QE on the chip.

The most important point in these curves is the classical triangular shapes indicating that the spectral shape is identical for the two spectrometers and a uniform QE as a function of the wavelength. Effectively, the photodiode current is proportional to the photon flux while the light power is inversely proportional to $h \nu / \lambda$. Therefore, the resulting ratio is proportional to $\lambda$. 
Note that we added a measurement in IR domain with a $3-5 \mu \mathrm{m}$ filter. This is to overcome the interferences issue, and so make QE measurements in IR domain with and without interferences.

QE extractions have been then performed in visible and IR range. Results are shown on Figure 4.

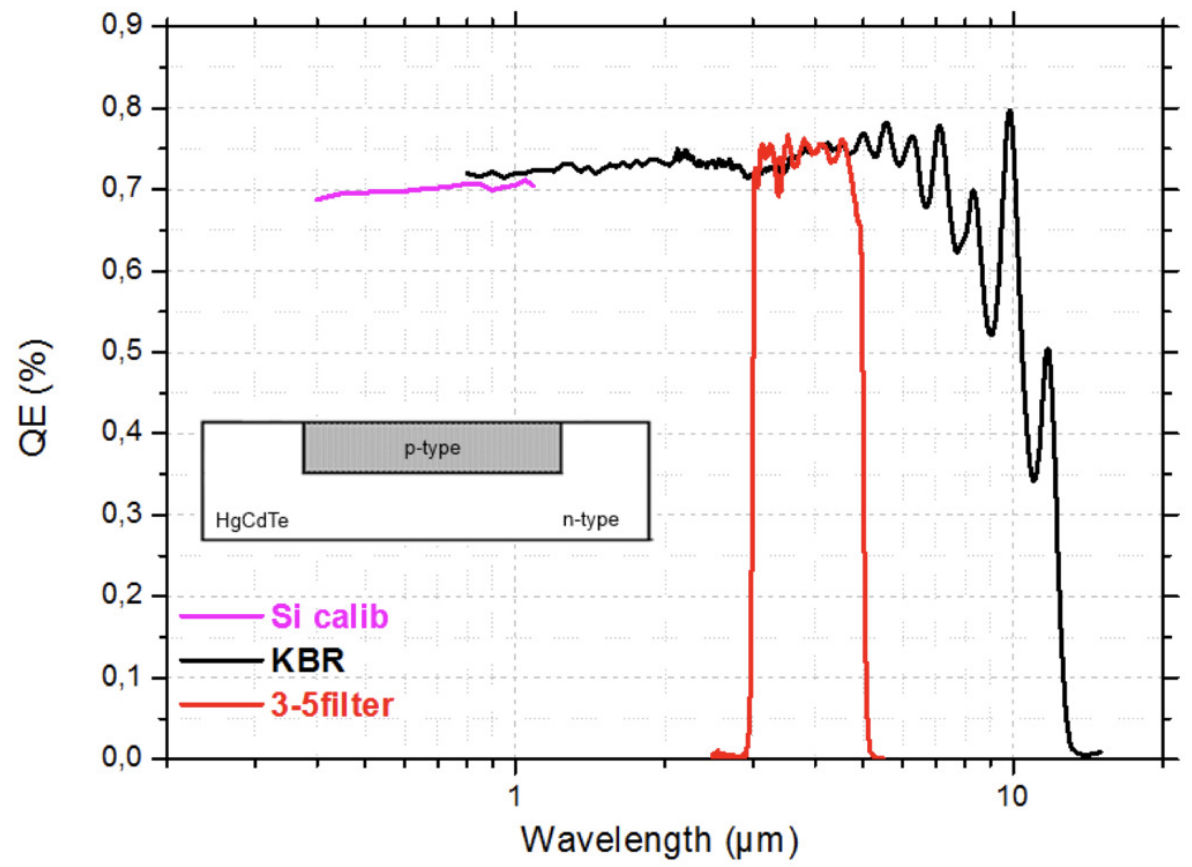

Figure 4. Spectral QE obtain in visible, 3-5 $\mu \mathrm{m}$ and total IR domain

As we can see, all measurements are consistent with each other. A difference of about $4 \%$ is observed between IR and visible (reference measurement) extraction, demonstrating the good accuracy of the two set-ups. We are now capable to calibrate $\mathrm{HgCdTe}$ device on IR domain without substrate removal. Thus, to deliver an operational detector at CEA IRFU, we decided to perform the calibration only in the IR range. The aim is to eliminate the interferences for the long wavelengths. The $\mathrm{QE}$ as a function of the temperature is shown on figure 5. The device consists of $8030 \mu \mathrm{m}$ pitch diodes in parallel to increase the detection surface. Moreover, we extract the dark current from $80 \mathrm{~K}$ to $50 \mathrm{~K}$ in order to give a complete tool to choose the most effective condition as a function of the needs (operating temperature and flux condition). 

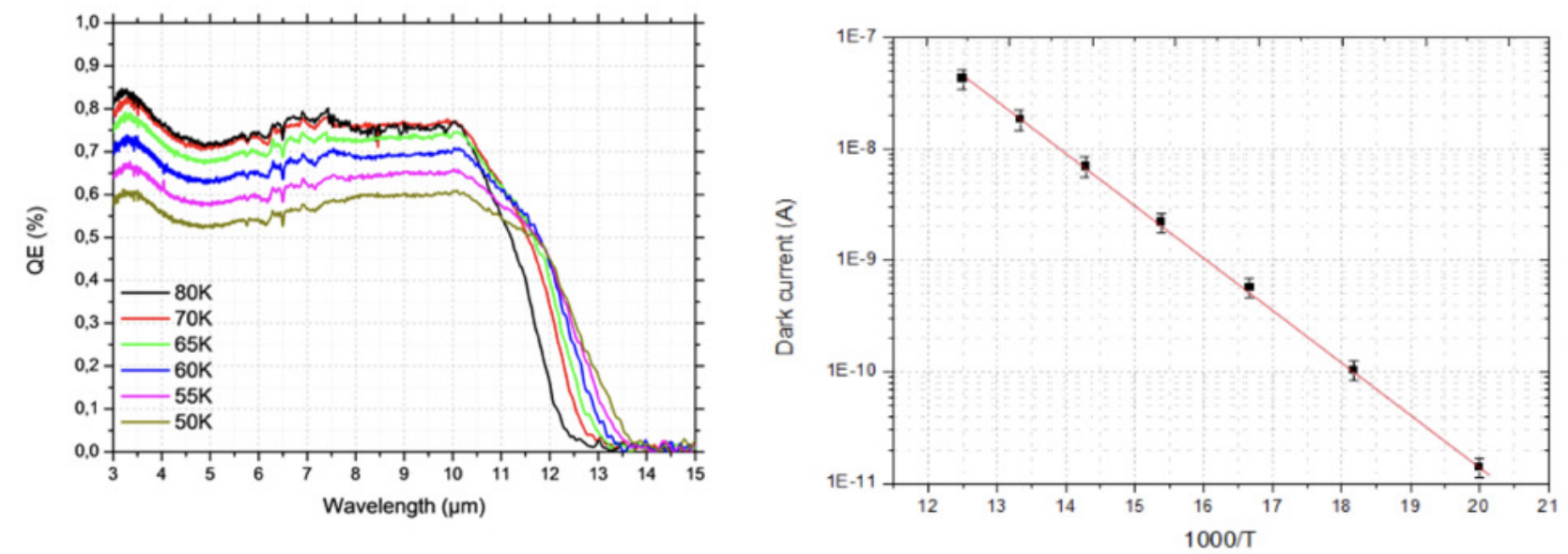

Figure 5. QE and dark currents as a function of temperature for 80 diodes in parallel

The QE is very good, around $75 \%$ at $80 \mathrm{~K}$. Nevertheless, a slight decrease is notable below $60 \mathrm{~K}$. This phenomenon has been already observed on p-on-n architecture. ${ }^{6}$ This is due to the fact that the diffusion length (capability to the electronic charge to diffuse into $\mathrm{HgCdTe}$ material) decreases strongly at low temperature. The consequence of this is a decrease of the fill factor of the photodiode and so a decrease of the photoresponse. However, QE values stay at a high level close to $60 \%$ at $50 \mathrm{~K}$.

A specific ceramic board has been developed to match electrical interfaces of the device with the CEA-IRFU set-up (figure 6).

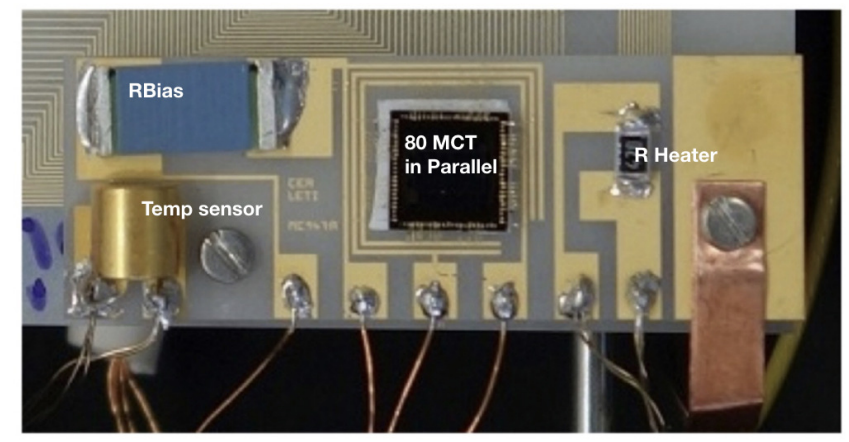

Figure 6. The calibrated photodiode mounted on its ceramic board.

\section{CRYO-HEMT CURRENT AMPLIFIER}

The readout of the calibrated MCT chips requires a transimpedance amplifier. However, the distance between the MCT chip located inside the FP and the connector at room temperature is roughly $2 \mathrm{~m}$. Consequently, the cables act as antennas, and make very difficult the readout of $<\mathrm{nA}$ current. The first tries using even a fA $/ \sqrt{H z}$ current amplifier at room temperature was not persuasive. To reduce the cable length between the MCT chip and the readout, the development of a dedicated cold amplifier is mandatory. As we know, off the shelf components are not suitable for $<60 \mathrm{~K}$ applications: to achieve such a cold amplification, we chose Cryo-HEMT transistor 
developed by $\mathrm{C} 2 \mathrm{~N}$ for its low noise and low operating temperature.

There are few available high impedance transistors technologies when dealing with low temperature electronics. In the past decades, cryogenic transistors with low noise and high input impedance were based on silicon Junction Field-Effect Transistor (JFET). However, their operating temperature is limited to above 100K due the charge freeze-out. This could be an issue for our application because the cable length between the MCT and the 100K stage is still quite long. The other two types of FET are MOSFET and Cryo-HEMT. The MOSFET suffers from an extremely high low-frequency noise, which is not suitable for our DC photonic current. The Cryo-HEMT is based on a 2DEG (Two Dimensional Electron Gas) with High Electron Mobility, insuring a very low working temperature (proven down to $100 \mathrm{mK}$ ). The CNRS/C2N laboratory has developed specific Cryo-HEMT using AlGaAs/GaAs heterojunctions, ${ }^{7}$ it has demonstrated that the input noise is inversely proportional to the square root of the input capacitance. Different types of Cryo-HEMT with different gate geometries were designed and manufactured. We have used Cryo-HEMT with an input capacitance of $92 \mathrm{pF}$, they have a typical voltage noise of $0.46 \mathrm{nV} / \sqrt{H z}$ at $1 \mathrm{kHz}$, the current noise is about a few $\mathrm{aA} / \sqrt{H z}$, which is negligible.

In the past years, we have used these Cryo-HEMTs to develop a generic voltage amplifier, that could be easily adapted to different kinds of applications. ${ }^{8}$ This amplifier board has been tested at $4.2 \mathrm{~K}$ and has demonstrated a gain of 50 , a power consumption of $1.5 \mathrm{~mW}$ per channel, and an input noise voltage lower than $0.5 \mathrm{nV} / \sqrt{H z}$ at $1 \mathrm{kHz}$, which is very close to noise performance of the Cryo-HEMT themselves. Here the idea is to take full advantage of this previous development and adapt this board as much as possible in order to make a current amplifier for the calibrated MCT. As discussed earlier, the calibrated MCT operating temperature is between 40 and $60 \mathrm{~K}$. Because the voltage noise of the Cryo-HEMT is proportional to its temperature, and because such a temperature was available in the setup, we chose to put the Cryo-HEMT board at the $4 \mathrm{~K}$ stage to get better noise performance. In this case, the cable length between the MCT chip to the amplifier board is only $15 \mathrm{~cm}$. The voltage amplifier board is a 4-channels Cascode two-stage amplifier. To make a current amplifier, the first idea - the not chosen option - was to use feedback resistance that connects the Cryo-HEMT3 source to the Cryo-HEMT2 gate. This design keeps the voltage across the photodiode constant, and the current is read via the feedback resistance Rf. However, this is a not working solution because the photonic current being in DC, the voltage across the feedback resistance should be at least $400 \mathrm{mV}(\mathrm{Vsg}=\mathrm{Vds}=100 \mathrm{mV})$ to maintain the bias point of the transistors. Considering only the dark current of $1 \mathrm{nA}$, and $\mathrm{Rf}=10 \mathrm{M} \Omega$, the voltage across $\mathrm{Rf}$ is $\mathrm{VRf}=10 \mathrm{mV}$, which is not sufficient. The VRf could be increased by adding a constant offset current to the photonic one, however this solution requires an additional bias source, and will also increase the noise by a factor of $\sqrt{2}$. Therefore, we have decided to explore a simpler scheme without using a feedback resistance (figure 7 ). 


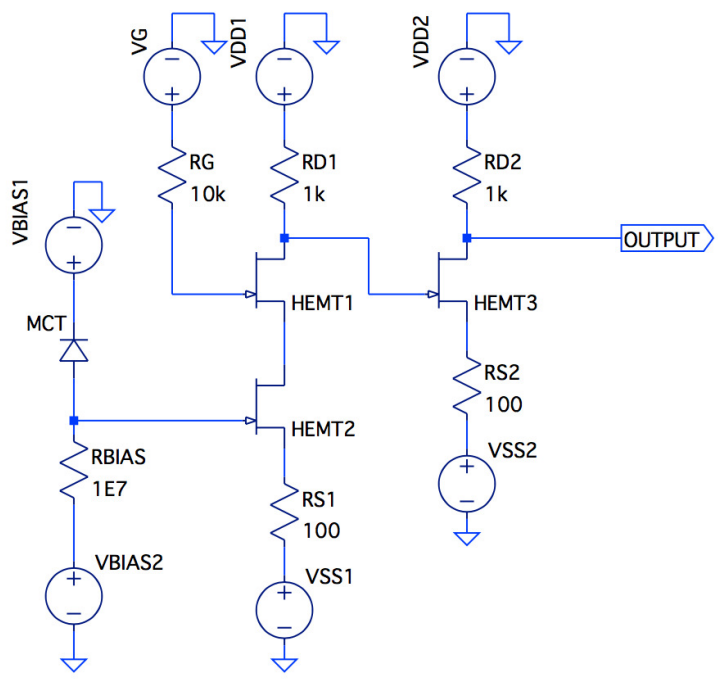

Figure 7. Transimpedance amplifier using only Cryo-HEMT transistors. To reduce the power consumption at low temperature, five voltage sources are used to bias the Cascode. The photonic current is converted to voltage using RBIAS. It uses the fact that the voltage variation across the MCT is very small when it biased in the so called plateau area (figure $7)$. The transimpedance gain is given by $R B I A S *(R D 1 * R D 2 / R S 1 * R S 2)$. The bandwidth is $1 / 2 \pi R_{B I A S} * C_{g s}$, where $C_{g s}$ is the input Cryo-HEMT capacitance (including parasitic capacitance)

When observing a typical IV curve of the diode, we note that we could make a full advantage of the so-called plateau area, when the current is almost constant for a given flux. In our case, we can tolerate a voltage variation of up to $30 \mathrm{mV}$ without significant photonic current variation. Using this advantage, we simply convert the current to a voltage using a bias resistance $R_{B I A S}$ and we read the potential difference across $R_{B I A S}$ by the Cryo-HEMT voltage amplifier. Considering $R_{B I A S}=10 \mathrm{M} \Omega$, the current variation limit for a linear response is $3 \mathrm{nA}$. This is sufficient during a monochromatic scan because it induces a current variation of $0.1 \mathrm{nA}$ for about $10^{6} \mathrm{photon} / \mathrm{s} / \mathrm{pix}$ (and $\mathrm{QE}=0.65$ ). Because the monochromator is located at room temperature, we are mainly dominated by the $300 \mathrm{~K}$ thermal radiation. This constant flux level can be subtracted by adjusting the voltage source $V_{B I A S 2}$.

From the noise point of view, the equivalent input noise of the amplifier is the quadratic sum of all noise sources contributions: Cryo-HEMT voltage and current noise, and the $R_{B I A S}$ thermal noise. As discussed earlier, the current noise is negligible, the Johnson noise is about $46 \mathrm{nV} / \sqrt{H z}$ (at $4 \mathrm{~K}$ ). Thus, we are dominated by the Cryo-HEMT voltage noise at DC, which is close to $0.1 \mu V / \sqrt{H z}$. This voltage noise is transformed to current noise by the $R_{B I A S}$ resistance. Hence, we estimate the equivalent input current noise of this transimpedance amplifier to $0.1 \mu \mathrm{V} / 10 \mathrm{M} \Omega=10 \mathrm{fA} / \sqrt{H z}$. 


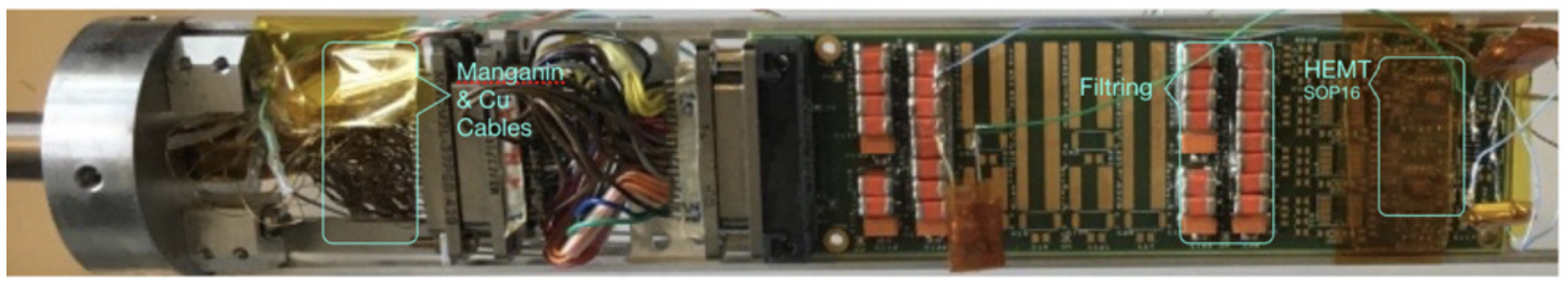

Figure 8. Cryo-HEMT-based voltage amplifier board mounted in its cryogenic stick. A temperature sensor from Lakeshore is also used

Cryo-HEMT transistors are not designed for room temperature. Therefore, there is a leakage current by transistors gates, which makes adjusting the bias working point very difficult. Furthermore, only low gain (between 1-2) could be obtained due to their low transconductance (gm). This is clearly an issue because it makes tests at $300 \mathrm{~K}$ not so obvious. On the other hand, the cryo-cooler cryostat dedicated to electro-optical characterization requires about 15 hours to reach $4 \mathrm{~K}$, and 3 days to warm up. This is also problematic because the first tests of Cryo-HEMT amp need several thermal cycles for debugging. Therefore, we have developed a cryogenic stick in order to perform tests in He dewar (figure 8). The stick is made of two parts: a stainless steel tube to insure good thermal gradient between 4.2 and $300 \mathrm{~K}$, and a mechanical support $4 \mathrm{~cm}$ wide that handles the amplifier board. The electrical wires have been carefully chosen ensuring a good compromise between electric resistance while optimizing the heat load. The board is supplied by batteries only when reaching the ultimate noise performance of the amplifier. To prevent batteries discharges during several hours of experiment, we have used a low noise high stability power supply from iTest. It has an output noise less than $5 \mathrm{nV} / \sqrt{H z}$ at $1 \mathrm{kHz}$. The noise characterisation is presented in figure 9. 


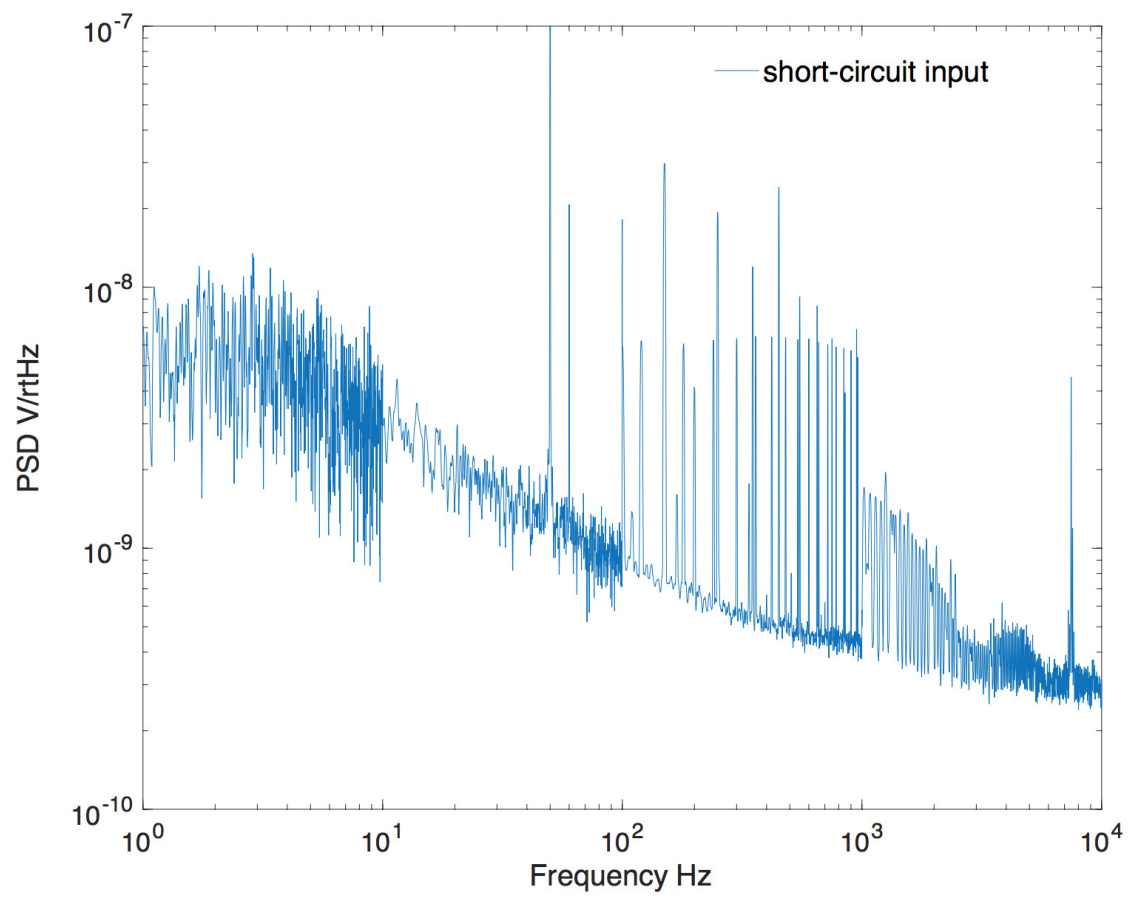

Figure 9. Equivalent input noise measurement of the amplifier board at $4.2 \mathrm{~K}$ with a short circuit at its input using batteries supplies. The noise level is similar to the Cryo-HEMT itself.

\section{EXPERIMENTAL SETUP AND RESULTS}

\subsection{Experimental setup}

The detector to be characterized (detector under test or DUT) and the calibrated detector are operated in a large test cryostat which was developed mainly for measurements of very low dark currents (top image in Figure 10). For this reason, the cryostat has no entrance window. Both detectors are placed inside a common housing which is light tight and thermally linked to the $4 \mathrm{~K}$ stage of the cryogenic machine. This way, the detectors are in an environment at about $8-10 \mathrm{~K}$ which generates no detectable parasitic photon flux in the spectral range of the detectors.

The QE measurement uses a monochromator (from Horiba Jobin Yvon) at room temperature which is optically coupled to an optical fiber. The fiber enters the cryostat through a vacuum feedthrough and is connected to the detector housing (Right image in Figure 10). Since one tip of the fiber is at room temperature, the fiber receives the $300 \mathrm{~K}$ radiation through its acceptance cone and propagates it all the way to the detector. At the level of the detector under test, the $300 \mathrm{~K}$ radiation generates a current of about $3 * 10^{7} \mathrm{e}-/ \mathrm{s} / \mathrm{pixel}$. For this reason, a small cryogenic shutter is also placed inside the detector housing to mask-unmask the tip of the fiber for dark current measurements. In order to remove the $300 \mathrm{~K}$ component of the photon flux received by the detectors, a shutter is placed as close as possible to the infrared emitter of the monochromator, and differential measurements are performed with the shutter open (300K + monochromatic radiations) and closed (300K radiation only). 

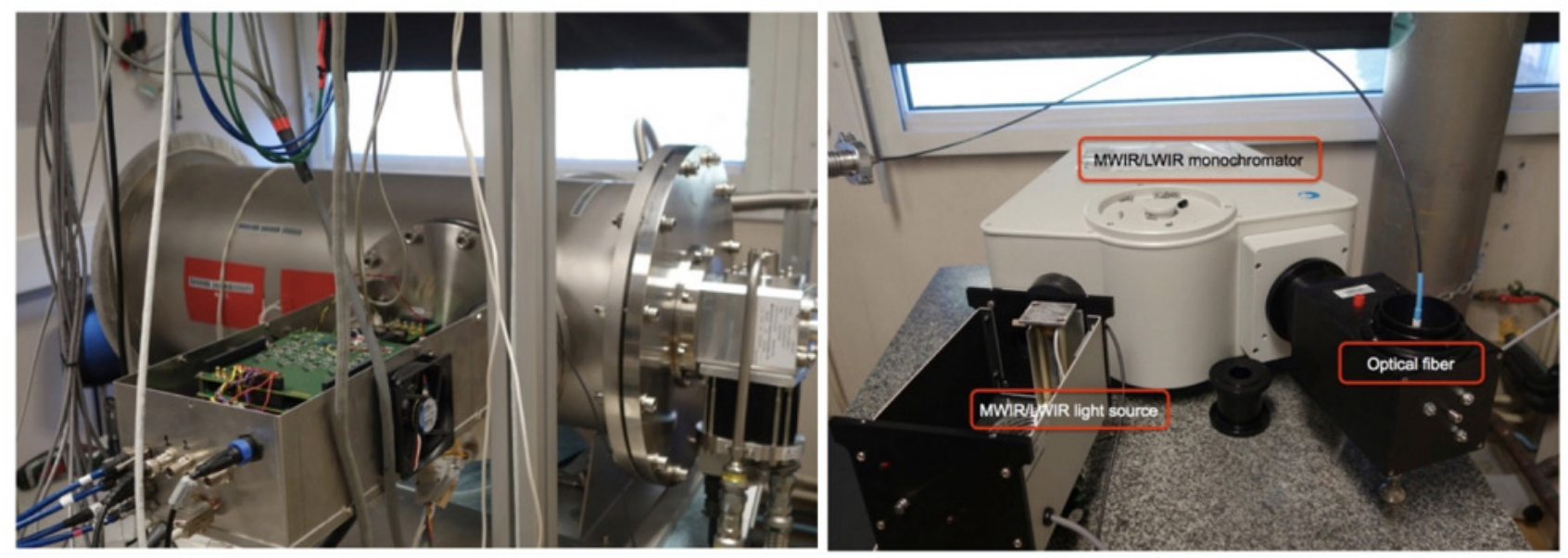

Figure 10. Test setup used for the characterization of the LWIR detectors. Left: the cryostat, with one of the front end electronics connected. Right: the monochromator and the optical fiber to bring the light inside the cryostat.

The detector under test is mounted onto a ceramic board which manages all the interfaces (electrical, thermal and mechanical) to the cryostat. The calibrated photodiode is mounted onto a daughter board of the main ceramic (Figure 11). The two detectors are independently thermally regulated. While the DUT is characterized from 20 to $80 \mathrm{~K}$, the calibrated photodiode is maintained at $60 \mathrm{~K}$.

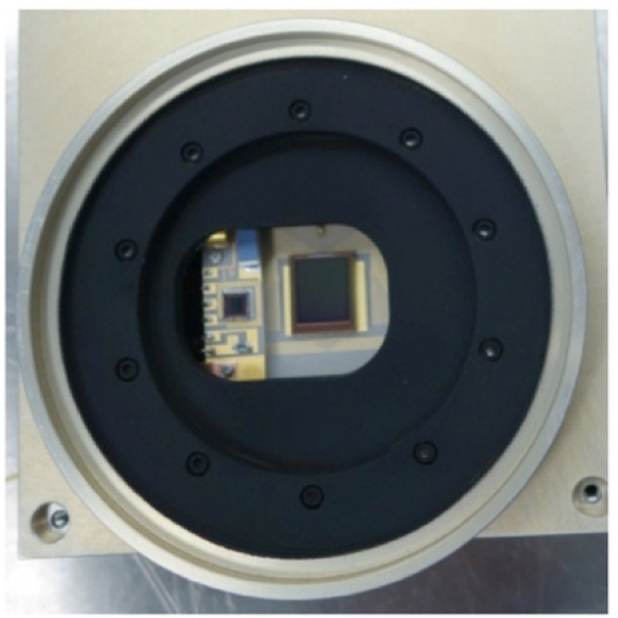

Figure 11. The detector under test (center of image) and the calibrated photodiode (to the left of the detector) inside the detector housing

Opposite to the detectors in the detector housing, and located above the center of the DUT, a small black body (with known emissivity) can be used to illuminate the DUT. Provided the spectral range of the DUT is known (for example using a monochromator scan), this simple geometry allows one to estimate the photon flux emitted by the black body from radiometry considerations. It is therefore possible to estimate the QE of the DUT using the black body and a relative spectral response.

The IV curves of the calibrated photodiodes in dark and under black body flux are shown in figure 12. From the so called plateau area, we have estimated the optimum bias voltage to $20 \mathrm{mV}$. As expected, the measured dark current is about $12.5 \mathrm{pA}$ (per photodiode) at $60 \mathrm{~K}$. 


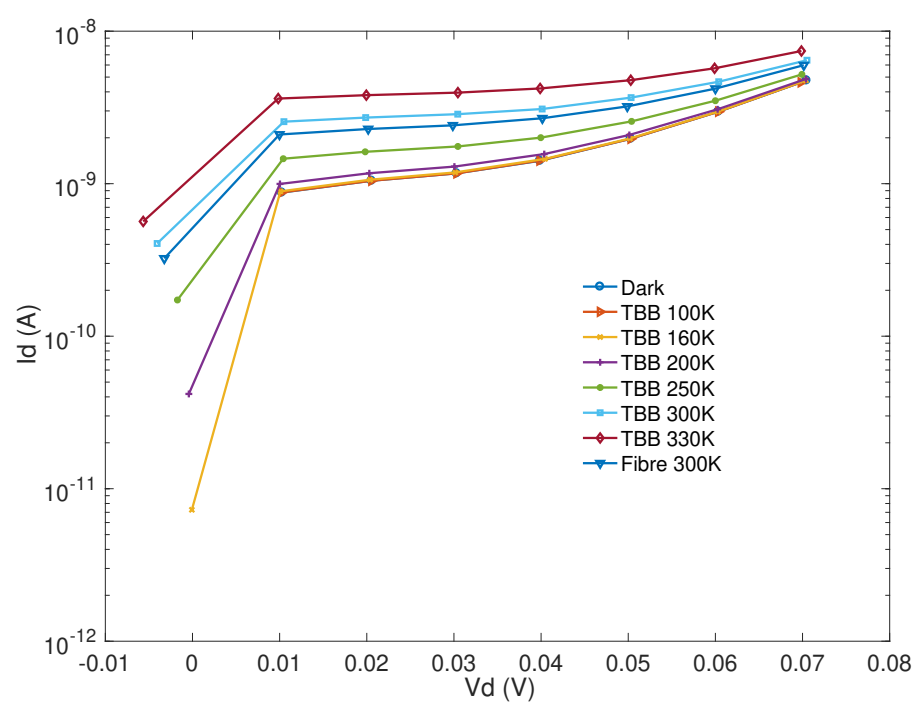

Figure 12. IV curve at dark and under photon flux for $80 \mathrm{MCT}$ at $60 \mathrm{~K}$, where TBB is the temperature of the Black body.

\subsection{Quantum efficiency measurements using the calibrated photodiode}

Both the DUT and the calibrated photodiodes were operated at $60 \mathrm{~K}$. The monochromator was used to scan the spectral range of the detector under test from 3 to $14 \mu \mathrm{m}$ in steps of 50nm. At each step, two measurements were performed, with the monochromator shutter open and closed in order to remove the contribution from the $300 \mathrm{~K}$ radiation. Each measurement of the DUT contained 10 readouts (Figure 13 left). The photodiode was also read at each step (Figure 13 right).
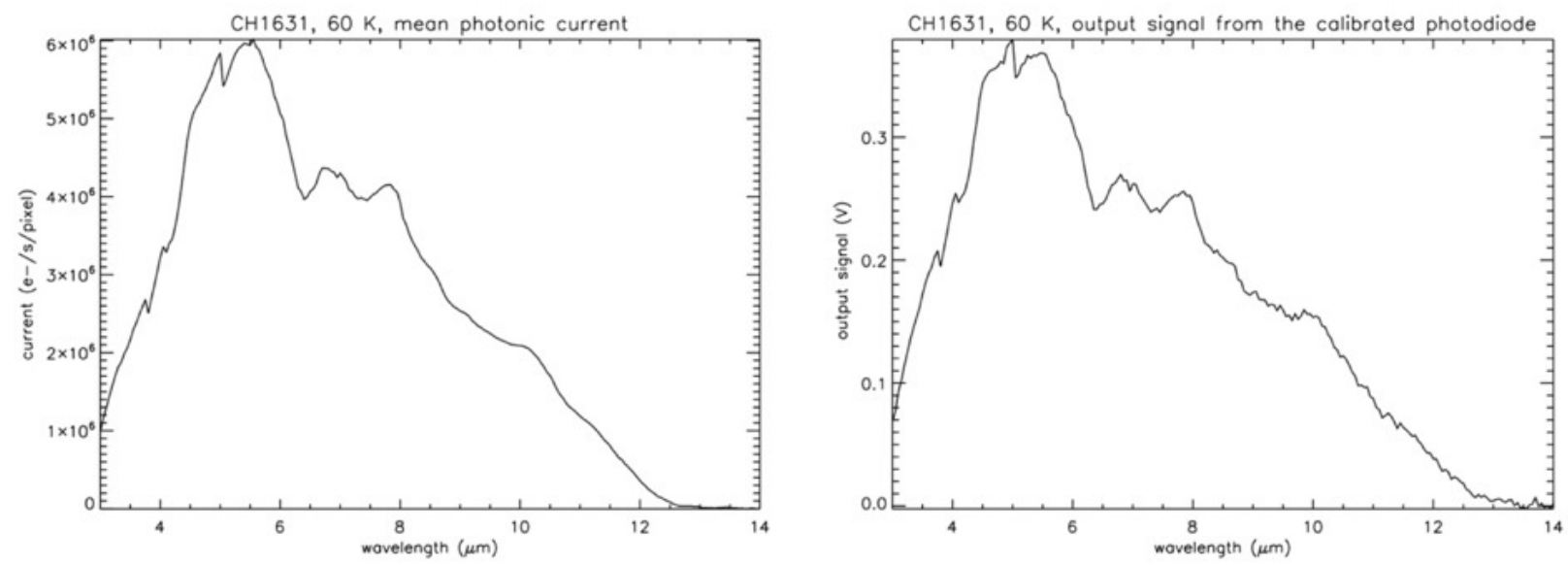

Figure 13. Spectral scan from 3 to $14 \mu \mathrm{m}$ : photonic current measured by the detector under test, averaged over all pixels (left) and output signal of the calibrated photodiode and cold amplifier (right).

The accuracy of the measurements is very good, with relative errors lower than $1 \%$ all the way to $12 \mu \mathrm{m}$. Beyond this wavelength, the QE of both the DUT and the photodiode get very small, resulting in low currents but the relative errors stay below $10 \%$ between 12 and $13 \mu \mathrm{m}$. Knowing the gain of the transimpedance amplifier (section 3), one can derive the photonic current delivered by the photodiode. The size and number of individual 
diodes making the calibrated photodiode are known (section 2). The ratio of illuminations between the center of the DUT and the location of the calibrated photodiode was measured at 0.4 , by placing the reference detector at the location of the DUT. Using all these data and the two spectral responses shown in Figure 13, we can derive the ratio of the spectral responses of the DUT and the reference detector. Finally, the quantum efficiency of the DUT is derived from the measured QE of the reference detector (section 2) by applying this ratio of spectral responses (Figure 14).

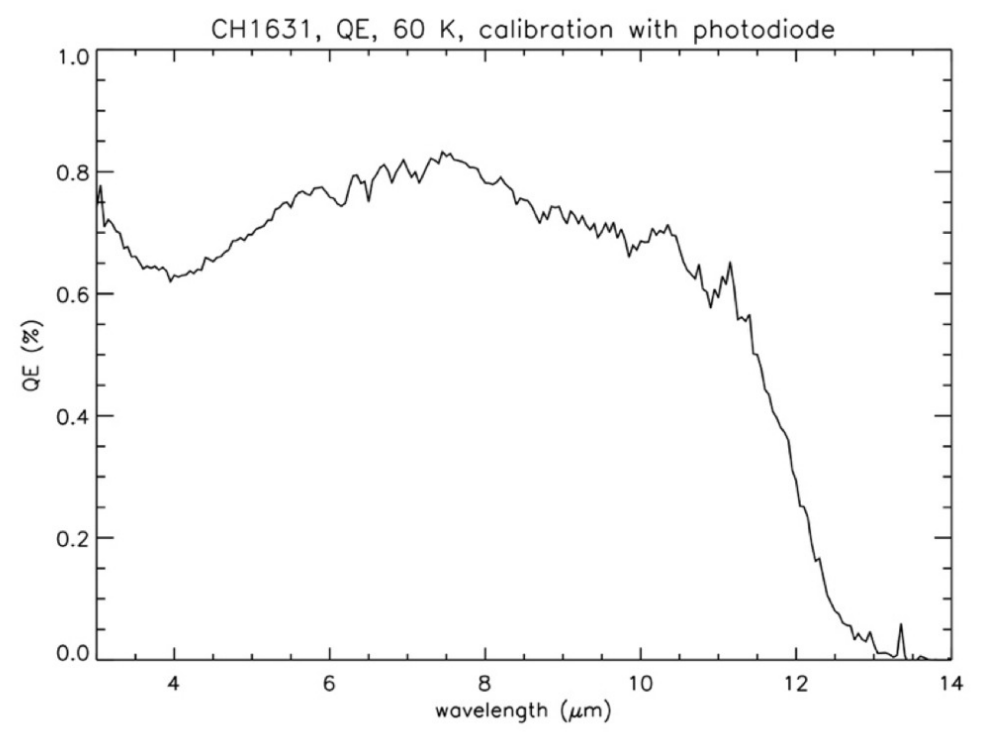

Figure 14. Quantum efficiency at $60 \mathrm{~K}$ of a Lynred $12.5 \mu \mathrm{m}$ cutoff LWIR device

\subsection{Comparison with quantum efficiencies using the internal black body}

The black body inside the detector housing can be used as an alternative way of estimating the quantum efficiency of the detector. Since there is no filter in the light path from the black body to the DUT, the photon flux that the DUT receives from the black body can be estimated from radiometric considerations, integrating a Planck radiation law (taking into account the emissivity of the black body) over the full spectral range of the detector and the solid angle under which a detector pixel sees the black body. The spectral range of the DUT is given by the monochromatic scan. The temperature of the black body is regulated and can be set anywhere between 30 and $340 \mathrm{~K}$. The computation of the photon flux emitted by the black body is strongly dependent on the maximum wavelength of absorption of the detector: the emission of a $250 \mathrm{~K}$ black body peaks at $12 \mu \mathrm{m}$, which means that, even though the QE becomes very small at wavelengths greater than $12.5 \mu \mathrm{m}$, the photon flux that can be absorbed is not negligible (figure 15). For example, about $9.2 \%$ of a $250 \mathrm{~K}$ black body radiation, integrated from 0.8 to $14.0 \mu \mathrm{m}$, is emitted in the range 13.5 to $14.0 \mu \mathrm{m}$, and about $4.6 \%$ is emitted in the range 13.75 to $14.0 \mu \mathrm{m}$. 


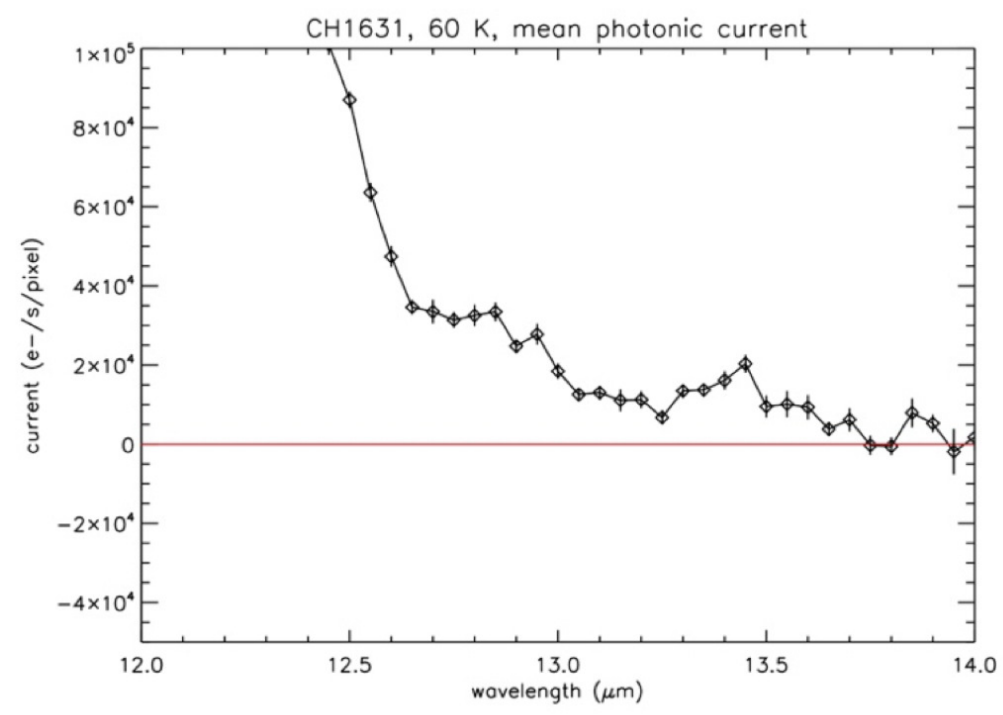

Figure 15. Zoom on the absorption of the detector beyond the cutoff wavelength. It is not obvious to measure where the absorption falls to 0 , which induces an uncertainty in the photon flux emitted by the black body, and therefore the quantum efficiency of the detector.

From there, a measurement of the current delivered by the DUT under illumination from the black body gives the QE averaged over the full spectral range of the detector. In this case, one finds an average QE of $58 \%$, assuming the detector absorbs all the way up to $14.0 \mu \mathrm{m}$. This average value is then used to scale the relative QE derived as described above. At $60 \mathrm{~K}$, the QE averaged over the 8.5 to $12.0 \mu \mathrm{m}$ range is $63.3 \%$ using the calibrated photodiode and $62.6 \%$ using the black body's calibration. The two measurements are in excellent agreement, but again the latter one strongly depends on the proper measurement of the spectral range of the detector.

\section{CONCLUSION AND PERSPECTIVES}

One of the main electro-optical parameter of a semiconductor detector is its quantum efficiency, which measures the efficiency of the detection of the incident photons. The main difficulty is to measure the flux of photons reaching the detector. While it is fairly straightforward to do this in the visible range, thanks to calibrated Silicon photodiodes commercially available, it is much more difficult to do in the mid to long wave infrared range, as no reference detector are available.

CEA-LETI developed and calibrated such reference devices, using HgCdTe photodiodes from test vehicles built in previous detector development activities. In particular, we developed a calibrated photodiode in the LWIR range up to $12.5 \mu \mathrm{m}$. In parallel, CEA-IRFU developed a cold amplifier based on the use of Cryo-HEMT transistors to drive the output current of the calibrated photodiode outside of the cryostat for a clean measurement. The calibrated photodiode coupled to the cold amplifier allowed the measurement of the quantum efficiency of a LWIR $12.5 \mu \mathrm{m}$ cutoff detector built by Lynred in the frame of a ESA funded detector development activity.

The LWIR photodiode developed in this work will be used to measure the quantum efficiency of prototype detectors for the science instrument of Ariel (ESA's M4 mission) that are being manufactured thanks to funding from both CNES and ESA. Calibrated photodiodes optimized for other wavelength ranges are also being developed. The next ones will use avalanche $\mathrm{HgCdTe}$ photodiodes at $3.3 \mu \mathrm{m}$ cutoff wavelength to measure the quantum efficiency of short wave infrared detectors (2.1 and $2.5 \mu \mathrm{m}$ cutoff) developed in the frame of one ESA and one EC (H2020) programs. They will be coupled to cold Cryo-HEMT amplifiers identical to the ones developed in this study. Last, calibrated photodiodes optimized for the mid wave infrared will also be manufactured. 


\section{ACKNOWLEDGMENTS}

This work was performed in the frame of ESA contract no. 4000113066/15/NL/RA.

This work has been partially supported by the LabEx FOCUS ANR-11-LABX-0013.

\section{REFERENCES}

[1] Boulade, O., Baier, N., Castelein, P., Cervera, C., Chorier, P., Destefanis, G., Fièque, B., Gravrand, O., Guellec, F., Moreau, V., Mulet, P., Pinsard, F., and Zanatta, J.-P., "Development and characterisation of MCT detectors for space astrophysics at CEA," in [Society of Photo-Optical Instrumentation Engineers (SPIE) Conference Series], Society of Photo-Optical Instrumentation Engineers (SPIE) Conference Series 10563, 105631W (Nov. 2017).

[2] Baier, N., Cervera, C., Gravrand, O., Mollard, L., Lobre, C., Destefanis, G., Bourgeois, G., Zanatta, J., Boulade, O., and Moreau, V., "Latest developments in long-wavelength and very-long-wavelength infrared detection with p-on-n hgcdte," Journal of Electronic Materials 44, 3144-3150 (Sep 2015).

[3] Gravrand, O. and Rothman, J., "Hgcdte quantum detection: from long-wave ir down to uv," Journal of Electronic Materials 40, 1781-1784 (Aug 2011).

[4] Baier, N., Mollard, L., Rothman, J., Destéfanis, G., Ballet, P., Bourgeois, G., Zanatta, J. P., Tchagaspanian, M., Courtas, S., Fougères, P., Pautet, C., Pidancier, P., and Rubaldo, L., "Status of p-on-n HgCdTe technologies at DEFIR," in [Infrared Technology and Applications XXXV], Proceedings of the SPIE 7298, 729823 (May 2009).

[5] Rothman, J., Meilhan, J., Perrais, G., Belle, J.-P., and Gravrand, O., "Maximum entropy mobility spectrum analysis of hgcdte heterostructures," Journal of Electronic Materials 35, 1174-1184 (Jun 2006).

[6] Cervera, C., Baier, N., Gravrand, O., Mollard, L., Lobre, C., Destefanis, G., Zanatta, J. P., Boulade, O., and Moreau, V., "Low-dark current p-on-n MCT detector in long and very long-wavelength infrared," in [Infrared Technology and Applications XLI], Proceedings of the SPIE 9451, 945129 (June 2015).

[7] Dong, Q., Liang, Y. X., Ferry, D., Cavanna, A., Gennser, U., Couraud, L., and Jin, Y., "Ultra-low noise high electron mobility transistors for high-impedance and low-frequency deep cryogenic readout electronics," Applied Physics Letters 105(1), 013504 (2014).

[8] de la Broïse, X. and Bounab, A., "Cryogenic ultra-low noise HEMT amplifiers board," Nuclear Instruments and Methods in Physics Research A 787, 51-54 (July 2015). 